\title{
Noninvasive assessment of liver steatosis using ultrasound methods
}

\author{
Monica Lupşor-Platon ${ }^{1}$, Horia Stefănescu ${ }^{2}$, Daniel Mureșan ${ }^{3}$, Mira Florea ${ }^{4}$, \\ Mária Erzsébet Szász ${ }^{1}$, Anca Maniu ${ }^{1}$, Radu Badea $^{1}$
}

${ }^{1}$ Department of Medical Imaging, Regional Institute of Gastroenterology and Hepatology „Prof. Dr. Octavian Fodor”, ${ }^{2}$ Department of Hepatology, Regional Institute of Gastroenterology and Hepatology „Prof. Dr. Octavian Fodor”, ${ }^{3} 1^{\text {st }}$ Department of Obstetrics and Gynecology, ${ }^{4}$ Diagnosis and Treatment Center, ,Iuliu Hatieganu” University of Medicine and Pharmacy, Cluj-Napoca, Romania

\begin{abstract}
Hepatic steatosis is a condition frequently encountered in clinical practice, with potential progression towards chirrhosis and hepatocellular carcinoma. Ultrasonography (US) is one of the noninvasive imaging techniques used in the diagnosis of steatosis. We will review the US diagnostic criteria, the US performance in the diagnosis and grading of hepatic steatosis, the US steatosis models, but also its limitations in the diagnosis of steatosis. In addition, we will discuss 2 modern methods of assessing hepatic steatosis using ultrasounds, namely the computerized processing of data forming the US image and the controlled attenuation parameter measured with unidimensional transient elastography.
\end{abstract}

Keywords: steatosis, noninvasive, ultrasonography, computerized processing, controlled attenuation parameter

\section{Introduction}

Hepatic steatosis (HS) summarizes a wide spectrum of conditions characterized by triglyceride accumulation within the cytoplasm of hepatocytes. It is more frequent in patients who drink alcohol on a regular basis, but also in those who are not consumers (nonalcoholic fatty liver disease - NAFLD) [1]. In fact, HS is the primary lesion of NAFLD, which, as a consequence of the worldwide burden of visceral obesity, is now the main cause of chronic liver disease (CLD) in western countries [2].

Although HS was traditionally regarded as a relatively inconsequential physical condition, its role in the pathogenesis of various clinically important diseases has

Received 24.06.2014 Accepted 04.07.2014

Med Ultrason

2014, Vol. 16, No 3, 236-245

Corresponding author: Monica Lupsor-Platon

Department of Medical Imaging, Regional

Institute of Gastroenterology and Hepatology

"Prof Dr Octavian Fodor"

19-21 Croitorilor Street

400162 Cluj-Napoca, Romania

E-mail: monica.lupsor@umfcluj.ro recently been increasingly recognized [3]. HS can progress to steatohepatitis, fibrosis, even cirrhosis and hepatocellular carcinoma in some patients [4-6]. On the other hand, steatosis is a well recognized co-factor accelerating the progression of CLD [7], it reduces the likelihood of sustained virological response in patients with HCV [8] and, in patients undergoing liver resection, HS independently increases the risk of postoperative complications and death [9]. The assessment of HS plays an important role in the preoperative evaluation of liver donors for transplantation $[10,11]$. Especially the macrovesicular subtype is critical for donor selection, since it has been associated with a greater risk of primary nonfunction after transplantation $[12,13]$.

HS is commonly observed in daily clinical practice and its prevalence is increasing in parallel with the pandemics of obesity and type 2 diabetes mellitus [14]. In a retrospective study performed on a hospital population of 14,009 patients, HS was found in $16.05 \%$ of cases using ultrasonography (US); steatosis was identified in $41 \%$ of all patients with diffuse liver diseases [15].

In histological terms, steatosis usually appears in the macrovesicular form and a lot less frequently in mi- 
crovesicular form. The microvesicular and macrovesicular forms can also coexist. When the former is present alone, particular causes should be sought: steatosis in pregnancy, Reye's syndrome and certain drug-induced steatosis [16].

The more precise method for the detection of steatosis is the liver biopsy (LB) [17]. Compared to it, the clinical examination and laboratory tests have a rather low positive predictive value [18]. However, LB is invasive and may result in severe complications [19]. Furthermore, it is also susceptible to potential sampling errors and cannot be readily repeated for adequate patient follow-up $[20,21]$. Therefore, there has been increased debate on wether LB is the only accurate diagnostic method, or if it can be, at least to some extent, substituted by imaging diagnostic methods. Of these, computed tomography exposes the patient to radiation, lacks sensitivity for small amounts of fat and is susceptible to intermachine variability [22]. Proton magnetic resonance spectroscopy and magnetic resonance imaging provide high accuracy in the quantification of liver fat, but are limited by low availability, high cost and lack of standardization [23].

Ultrasonography (US) remains an accessible imaging method, without side effects and thus is potentially very useful for long-term monitorization of disease progression. Indeed, conventional B-mode US is the most common technique used to assess the presence of fatty liver in clinical settings and population studies [24].

\section{Ultrasound diagnostic criteria for hepatic steatosis}

It is very important to avoid false positive results when searching for steatosis using US, because the US aspect of steatosis is not entirely specific [16].

Primarilly, the US changes in HS only become apparent at a hepatocyte fat accumulation above $15-20 \%$. These changes include: hepatomegaly in various degrees; hyperechogenicity of the parenchyma ("bright liver") due to the high number of highly reflective interfaces produced by increased intracellular fat deposition; ultrasound attenuation in subcapsular areas; difficult visualisation of portal venules, gallbladder walls and liver capsule (because the increased liver echogenicity leads to decreased acoustic impedance between the parenchyma and the portal venule walls); the apparent dilatation of vessels (especially hepatic veins) and biliary ducts; false transonic aspect of the right kidney as compared to liver parenchyma $[25,26]$ (fig 1).

According to some authors, the increased parenchymal echogenicity has a 0.87 positive predictive value for the steatosis diagnosis (confirmed by biopsy). When also considering the other diagnostic criteria stated above, the positive predictive value increases to 0.94 [27].

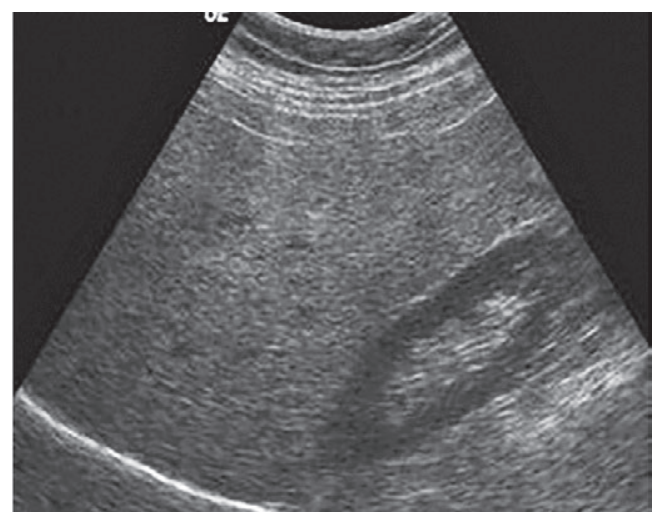

Fig 1. Liver steatosis. Hepatomegaly, hyperechogenicity of the parenchyma ("bright liver"), ultrasound attenuation in subcapsular areas, false transonic aspect of the right kidney as compared to liver parenchyma

\section{Quantitative assessment of hepatic steatosis}

The US evaluation of steatosis is mainly qualitative [28]. The qualitative grading is conveniently classified as mild, moderate or severe, or grade $0-3$ with 0 being normal [29]. Grade 1 (mild) is represented by a mild diffuse increase in fine echoes in the hepatic parenchyma with normal visualisation of the diaphragm and intrahepatic vessel borders. Grade 2 (moderate) is represented by a moderate diffuse increase in fine echoes with slightly impaired visualisation of the intrahepatic vessels and diaphragm. Grade 3 (marked) is represented by a marked increase in fine echoes with poor or no visualisation of the intrahepatic vessel borders, diaphragm and posterior portion of the right lobe of the liver [30].

\section{The US performance in detecting steatosis}

Generally, it is considered that US has $60-94 \%$ sensitivity and $88-95 \%$ specificity in detecting hepatic steatosis [18]. US sensitivity decreases at lower degrees of fatty load, with $80 \%$ sensitivity at fat accumulation above $30 \%$, as opposed to $55 \%$ when the fat content reaches only $10-19 \%$. In addition, in morbidly obese patients US sensitivity and specificity decrease to $49 \%$ and $75 \%$ respectively [30].

In a metaanalysis including 34 studies and 2815 participants [24], the overall sensitivity of US to discriminate moderate to severe histologically defined fatty liver from the absence of steatosis was $84.8 \%$ (95\% confidence interval [CI]: 79.5-88.9), the specificity was $93.6 \%$ (87.2-97.0), the positive likelihood ratio was $13.3 \%$ (6.427.6), the negative likelihood ratio was $0.16(0.12-0.22)$, 
and the summary area under the ROC curve was 0.93 (0.91-0.95).

Of the various criteria used to diagnose the presence of HS on US, the highest sensitivity and specificity for the presence of steatosis confirmed on histology is for macrovesicular steatosis $\geq 20 \%$ of the total liver area. With a greater area of fat deposition on histology, the sensitivity increased to $100 \%$ with only a small reduction in specificity [31].

In the study performed by Dasarathy et al [31], the hepatorenal echo contrast and liver brightness were able to identify the presence of hepatocyte fat accumulation $\geq 20 \%$ with a sensitivity of $96.4 \%$ and a specificity of $97.8 \%$. The criteria for vascular attenuation had lower sensitivity and specificity $(60.7 \%$ and $97.8 \%$ for portal vein blurring, respectively $92.9 \%$ and $95.6 \%$ for hepatic vein blurring). For the detection of the same amount of fat, the poor visualization of the diaphragm had 39.3\% sensitivity and $93.3 \%$ specificity.

Even though the sensitivity and specificity of hepatic vein blurring was higher than that of portal vein blurring, the combination of increased hepatic echogenicity and portal vein blurring was a more accurate predictor of hepatic steatosis. This was related to the high concordance between increased echogenicity and hepatic vein blurring (97.3\% agreement) so that adding hepatic vein blurring would not add any additional information. Hence, the combination of portal vein blurring and increased hepatic echogenicity was a better sonographic predictor [31].

In the metaanalysis by Hernaez et al, in studies where the accuracy of US parameters of fatty liver definition were evaluated individually, sensitivities of liver-to kidney contrast, vessel wall brightness, and deep beam attenuation in detecting moderate to severe steatosis (histologically defined) were 98\% (75\%-100\%), 81\% (70\%-89\%), and 59\% (45\%-72\%), respectively. The specificity was similar for all components (range 93\%95\%) [24]. The conclusion of this metaanalysis was that US is an accurate, reliable tool to detect moderate to severe steatosis, with sensitivity and specificity of $84.8 \%$ and $93.6 \%$, respectively [24]. On the other hand, the US performance in detecting lower steatosis grades is a lot weaker. In the study performed by Lee et al [3], US yielded low sensitivities for detecting all degrees of HS despite its relatively high sensitivities for moderateto-severe degrees of HS. This reconfirms the results of previous studies which reported low US sensitivity in the range of $28-67 \%$ for the diagnosis of $\mathrm{HS} \geq 3 \%$ or HS $\geq 5 \%$ [32,33]. In addition, US had a poor yield in the diagnosis of microvacuolar fat with an overall sensitivity of $43 \%$ and a specificity of $73 \%$ [31].
Factors influencing the US performance in detecting steatosis

In the study by Wang et al [34], the agreement rates between US and the histological findings were $74.3 \%$ in assessing the presence of steatosis and $61.4 \%$ in assessing steatosis severity. By using univariate analyses, body mass index (BMI) and necroinflamatory activity were associated with agreement between US and histology. The fibrosis score tends to influence the agreement between US and histology, almost reaching statistical significance $(\mathrm{p}=0.06)$. The age, sex, type of ultrasound machine and the status of viral hepatitis did not affect the agreement. By using multivariate analyses, the authors found that the fibrosis score, age and BMI were associated with agreement between US and histology. In fact, other studies had also found that several factors may affect the ability of US in diagnosing HS. For example, US in morbidly obese patients had the lowest accuracy and advanced fibrosis can reduce the sensitivity of US [30]. Regarding the relationship between age and the diagnostic accuracy of US, the possible explanation was that the aging process could change the echo texture of liver and/or kidney, and thus lead to a poor performance of US in older populations [34].

In the metaanalysis by Hernaez et al, when US was used to differentiate between the presence of histologically proven fatty liver alone and other pathological findings (such as hepatitis or fibrosis) or normal liver, the overall sensitivity was similar $(87.2 \%$; $95 \%$ CI: 77.8 93.0), but the specificity was substantially lower $(79.2 \%$; 95\% CI: 72.8-84.4). Correspondingly, the positive likelihood ratio was lower (4.2; 95\% CI: 3.3-5.4), but the negative likelihood ratio was unchanged $(0.16 ; 95 \% \mathrm{CI}$ : 0.09-0.28) [24].

Actually, liver fibrosis and inflammation may affect the diagnostic accuracy of US. A study with 118 biopsyproven NAFLD patients found that the sensitivity of US was $100 \%$ for detecting moderate to severe histological steatosis in patients with mild histological fibrosis. However, it decreased to $77.8 \%$ in those with advanced histological fibrosis [35]. Thus, advanced fibrosis can decrease the sensitivity of US in detecting moderate to severe histological steatosis because the advanced fibrosis could cause echogenic abnormalities on US.

In the study by Dasarathy et al [31], as well as in other studies [36], US was unable to discriminate between NASH and HS. This may be related to the NASH diagnostic criteria that require histological evidence of ballooning changes [37] as well as the low diagnostic accuracy of US in diagnosing fibrosis or inflammation. These data are consistent with previous reports that US 
is a sensitive method to diagnose HS, but not fibrosis or inflammation [27].

\section{Intra- and interobserver variability in the diagno- sis of HS}

Intra- and interobserver variability is one of the major issues occuring in the US diagnosis of HS, since it is based on fairly subjective criteria. In the metaanalysis by Hernaez et al, the range of kappa values for intra-rater evaluation was 0.54-0.92 and for the inter-rater evaluation was $0.44-1.00$ [24]. In the study by Lee et al, the performance of steatosis diagnosis through US differed quite significantly between 2 observers. For instance, for the diagnosis of hepatic steatosis $\geq 5 \%$, the sensitivity, specificity, positive and negative predictive values for the 2 observers were $61.7 \%$ vs $53.3 \%, 81.2 \%$ vs $93.1 \%$, $66.1 \%$ vs $82.1 \%$, and $78.1 \%$ vs $77.1 \%$ respectively [3]. For the diagnosis of hepatic steatosis $\geq 30 \%$, the differences occurred epsecially in steatose detection sensitivity. The sensitivity, specificity, positive and respectively negative predictive values for the 2 examiners were $81.8 \%$ vs $45.5 \%, 98 \%$ vs $98 \%$, $75 \%$ vs $62.5 \%$, and $98.7 \%$ vs $96.1 \%$ respectively [3]. In a retrospective US study, HS was evaluated by three independent, experienced radiologists in 168 patients and the examination was repeated one month later. The mean inter- and intra-observer agreement rates for the presence of increased liver fat were $72 \%$ and $76 \%$. Intra-observer agreement for severity of fatty liver ranged from $55 \%$ to $68 \%$ [38]. These results reveal that the method is highly dependent on the subjective impression of the examiner.

The high observer variability in US appears to be due to its inherent subjective nature as well as to the limitation of the traditional four-point visual grading system [36]. Although the grading system is widely used for US evaluation of HS [36], it may be too simplistic as it collapses a wide range of different degrees of HS into the same grade. The grading system also exacerbates observer variability by forcing the examiner to bluntly categorize similar degrees of HS that lie around the border between two adjacent grades into either grade [3].

\section{Doppler examination}

The Doppler examination found, in $43 \%$ of steatosis patients, abnormal flow in the hepatic veins (biphasic or monophasic flow), usually found in only $2 \%$ of healthy individuals. This particularity may be explained by the compression exerted by the enlarged hepatocytes (due to fat deposition) on the hepatic veins. The difference in hepatic vein flow in steatosis patients as compared to healthy subjects is statistically significant. No correlation was found between the degree of fatty infiltration and the flow pattern, nor between steatosis etiology and hepatic vein flow, but there is an increased tendency towards these changes in patients with hyperlipidemia [39]. A change in the hepatic vein flow may also occur in $50-73 \%$ of cirrhotic patients [40], due to the high fibrosis content leading to a decrease in parenchymal compliance.

The assessment of hepatic arteries reveals a significant decrease in the hepatic artery resistivity index in patients with severe steatosis, as compared to healthy individuals or patients with moderate steatosis [41].

\section{Ultrasonographic models of steatosis}

Steatosis may be found in various forms [42]: diffusse, multinodular, hypersteatosis (areas of relatively more steatotic areas of liver in patients with diffuse liver steatosis [43]), focal geographic steatosis, focal nodular steatosis, intralesional steatosis (in hepatic adenoma, hepatocellular carcinoma, regenerative nodules and rarely focal nodular hyperplasia [43-45]), perilesional steatosis (around metastases of insulinoma [42]), subcapsular steatosis (in patients with terminal renal illnesses and insulin-dependent diabetes mellitus under continuous peritoneal dialysis with insulin added to the peritoneal dialysate $[43,46])$ and periportal and perivenular steatosis.

Focal fatty infiltration usually appears geographic, but may be nodular and mass-like and, in this case, can result in a pseudotumor appearance (fig 2) $[42,47]$.

Focal steatosis is characterized by more or less extensive hyperechoic areas alternating with areas of normal echogenicity. The latter (called "focal spared areas") appear falsely hypoechoic as compared with the rest of the hyperechoic parenchyma, leading to confusion with hepatic tumors.

At Doppler examination, these hypoechoic areas do not distort normal hepatic vessels and do not have the

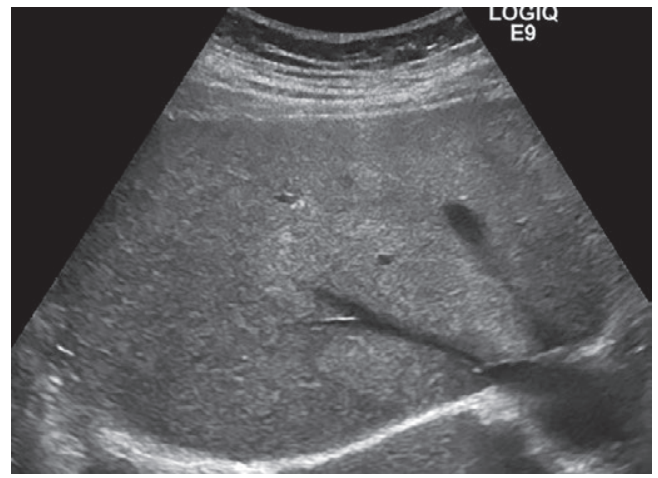

Fig 2. Focal steatosis. Hyperechoic areas around the hepatic veins 


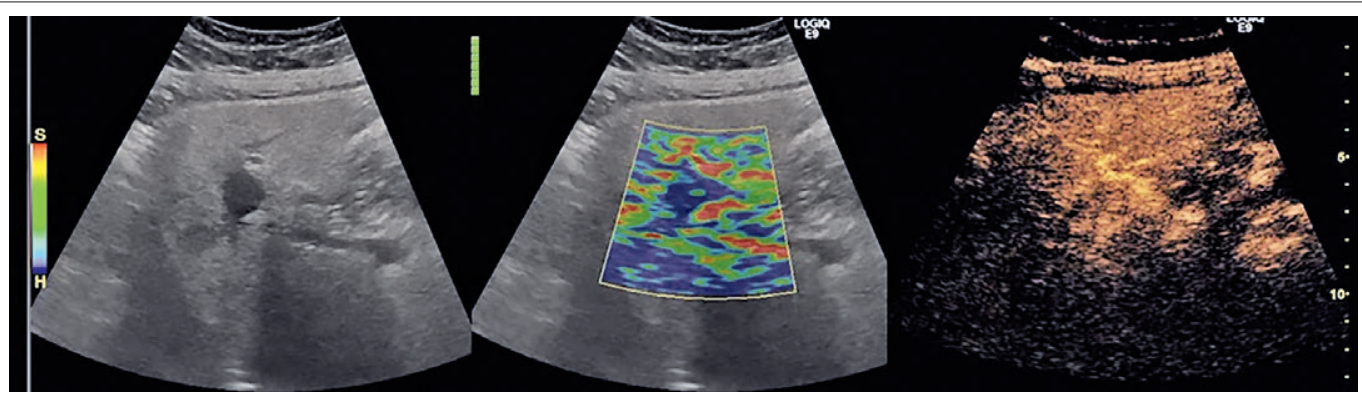

Fig 3. Hypoechoic nodular image in a steatotic liver. The aspect suggests a sparing area in a steatotic liver or a tumoral lesion. The elastography identifies a stiff lesion. On CEUS, enhancement in the periphery of the nodule, beginning in the arterial phase, with centripetal progression of the contrast agent, tipical for a hemangioma. Final diagnosis: hemangioma in a steatotic liver.

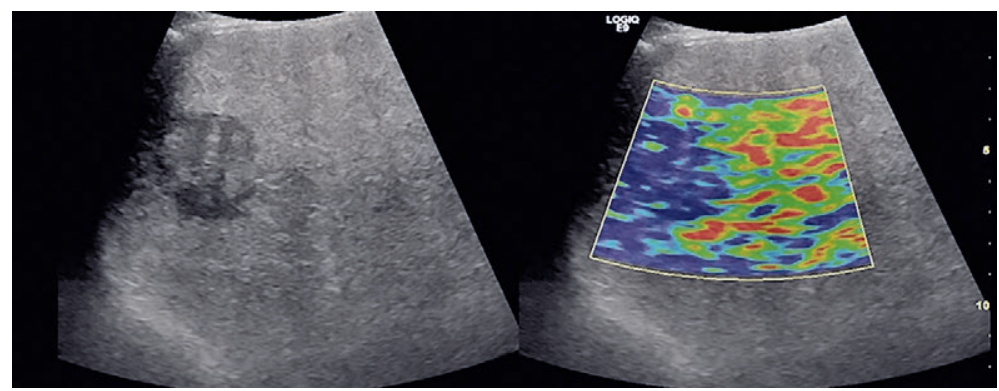

Fig 4. Round, hypoechoic, inhomogeneous image, with some echos within, located in a steatotic liver, investigated for the etiology. The structure appears on elastography to have significant stiffness. The final diagnosis was metastasis in a steatotic liver

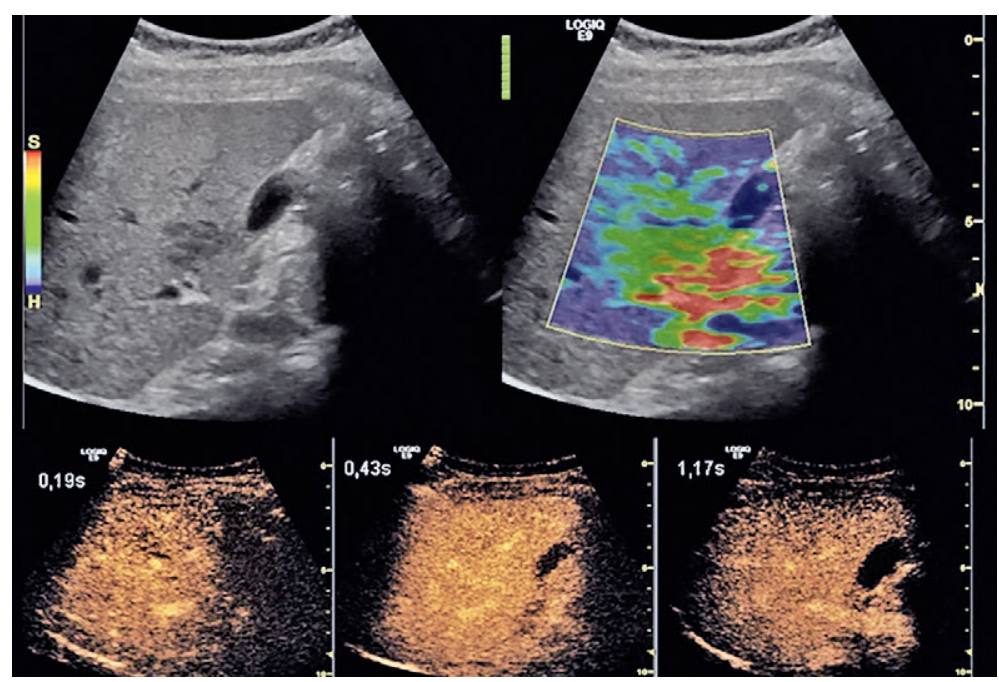

Fig 5. Focal sparing area in front of the portal vein. Hypoechoic image located anteriorly from the portal vein. The structure appears on elastography to have medium stiffness. On CEUS, the enhancement was homogeneous during the arterial, portal and parenchymal phases, without any apparent focal lesions. 


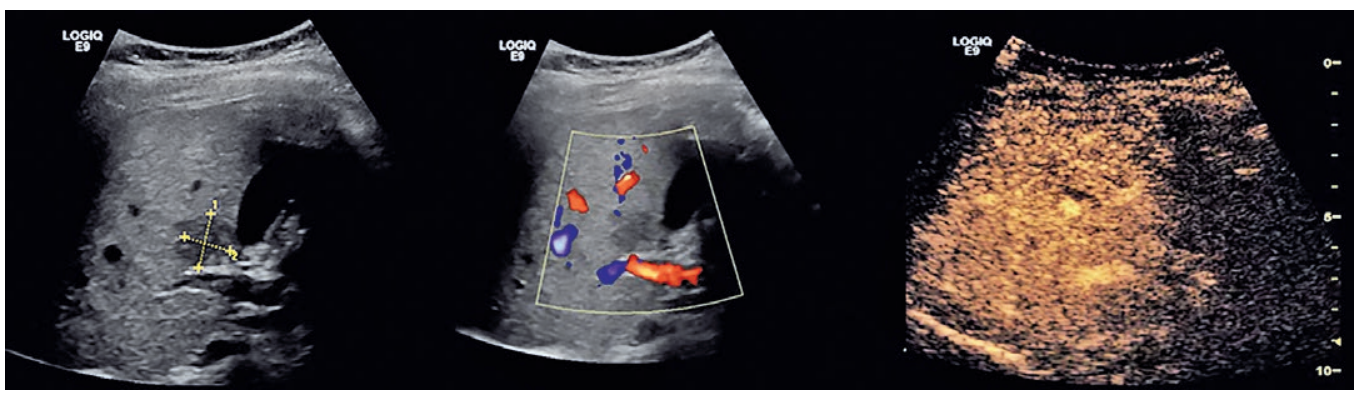

Fig 6. Focal sparing in the pericholecystic region. Hypoechoic image located around the galbladder in a steatotic liver. The Doppler examination cannot reveal any vessels within the lesion. On CEUS, the enhancement was homogeneous during the arterial, portal and parenchymal phases, without any apparent focal lesions.

hypervascular aspect of neoplastic tumors. Nevertheless, a clear differentiation from tumors is often difficult, requiring contrast enhanced US (CEUS), CT, MRI or even US-guided needdle biopsy [1] (fig 3, fig 4).

There are several types of hepatic fatty sparing: focal geographic sparing, focal nodular sparing, focal segmental or lobar sparing, perilesional sparing [42].

The fat-spared areas are most often found in the gallbladder bed and in the hepatic hilum, anteriorly from the portal vein bifurcation [48], but they can also be located around or distally from a tumor, due to the decrease in portal flow, arterio-portal shunts, hepatic venule compression around the tumor and/or the direct drainage of the arterial flow from the tumor in the neighbouring parenchyma [49] (fig 5).

In patients with a steatotic liver, Aubin et al [50] showed that usually fatty sparing occurs in segments IV and $\mathrm{V}$, surrounding the gallbladder (fig 6); this situation was found more frequently in patients who still had their gallbladder (78\%) than in cholecystectomised patients $(33 \%)$. The authors suggested a venous explanation, as there are almost always small cystic veins that drain directly into the liver and which are severed during cholecystectomy [16]

The pathophysiological explanation for the particular locations of these types of steatosis or focal sparing was supported by a theory concerning insulin, which stimulates the conversion of glucose to fatty acids [16]. About $30 \%$ of the vascular supply to the liver comes from the hepatic artery and about $70 \%$ from the hepatic portal vein. The insulin content of the tributaries of the hepatic portal vein is very variable. Left, or more frequently right gastric veins drain directly into the liver (mainly into segment IV), lowering the insulin concentration in that particular area which becomes a focal area of hepatic parenchyma which is less fatty than the rest of the liver, although the rest of the parenchyma is steatotic. Similarly, the superior branch of the pancreaticoduodenal arcade may drain not into the portal vein itself,

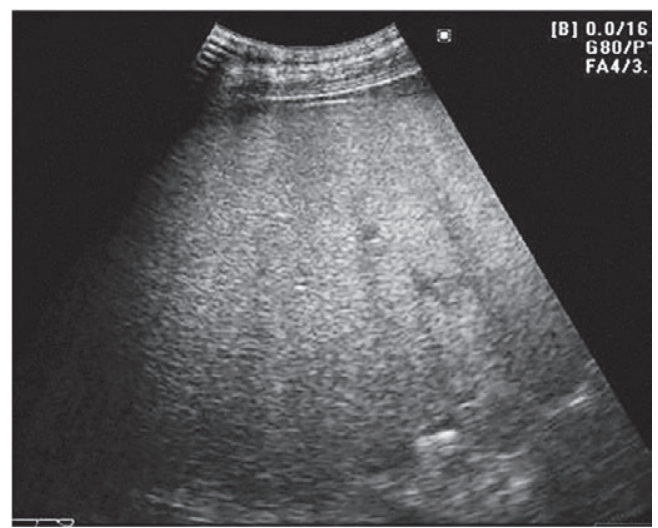

Fig 7. Liver with slightly higher echogenicity and moderate posterior attenuation, slightly inhomogeneous, without any focal lesions. On such an image the steatosis severity and the possible presence of fibrosis cannot be ascertained. The present image is usually described as ,steatofibrosis”.

but directly into the liver near the hilum, so that a localized area receives more insulin, increasing its fat content [16].

The increased echogenicity of the steatotic parenchyma may mask small hypo- or isoechoic tumors; this fact has the utmost importance especially during US check-ups in search of metastases in patients undergoing chemotherapy (certain chemotherapics may also induce steatosis) [51].

\section{The limitations of ultrasonography in diagnosing hepatic steatosis}

US has some limitations in diagnosing hepatic steatosis. The method cannot:

- differentiate between steatosis and steatohepatitis;

- establish with certainty the degree of fatty infiltration;

- differentiate between steatosis and other diffuse liver diseases characterized by increased echogenici- 
ty $[52,53]$. Of note, steatosis and fibrosis may have the same echographic appearance; for this reason, some authors use the term "steatofibrosis" in order to define the image obtained [18] (fig 7).

- always identify the spared or focal steatosis areas from hepatic focal lesions. In these cases, other imaging techniques (CEUS, CT, MRI) or even liver biopsy should be used.

\section{Optimization of the usual ultrasonographic examination through computerized processing of the US image}

One of the major problems of the usual US examination is the inability to differentiate between steatosis and fibrosis. Although they have a different pathologic basis, the main obstacle in discriminating between them is the lack of marked visual differences on the US image [54]. Attempts have been made to find ways of differentiating steatosis from fibrosis on the US image; some authors claim that only the fatty content increases the attenuation on the US image of cirrhosis patients [52], while others suggest that, in vitro, fibrosis also causes attenuation, but only half as much as fat [55]. Fibrosis may also be distinguished from fat by the "rougher" US texture it induces, as well as by the more definite outlining of the portal vein $[18,33,56]$. All these are, however, visual criteria, which depend upon the subjective interpretation of the examiner, limiting the reproducibility of the method and possibly leading to diagnostic errors. For these reasons, research is made to optimize the usual US image through the computerized analysis of the data making up the image.

The computerized processsing is based on the principle according to which the tissular changes occuring in some diseases (steatosis, chronic hepatitis, cirrhosis) induce physical and microarchitectural alterations (in density, viscosity, elasticity, homogeneity etc); these changes, although difficult to perceive visually, alter the propagation of ultrasounds and can therefore be identified through complex image analysis (ultrasonographic tissue characterization), as different from the normal texture pattern [57].

US tissue characterization may be performed through methods based on either the study of parenchymal echogenicity and ultrasound attenuation, or the quantification of textural parameters [57-60].

The majority of the studies used as a method for US image optimization the calculation of ,the sonographic hepatorenal index" (SHRI). The SHRI is the mean liver brightness divided by the mean renal cortex brightness. An image including both liver and kidney is required, typically showing segment VI of the liver and the upper

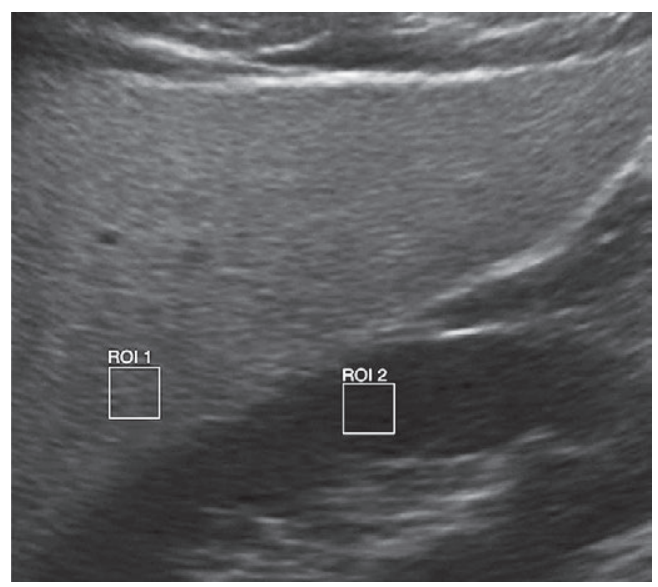

Fig 8. Quantification of echo intensity. Principle of the sonographic hepatorenal index. ROIs are placed over the liver parenchyma and renal cortex at the same depth. Mean echo intensities are determined on a standard US image. SHRI is calculated by dividing the mean liver brightness by the mean renal cortex brightness

pole of the right kidney [29]. Regions of interest (ROI) are selected in the liver parenchyma, excluding vessels, and in the renal cortex at the same field depth. The mean brightness of each ROI is determined using numerical values assigned to grey-scale pixels [29] (fig 8). Some ultrasound systems allow the placement of ROIs directly on the screen. Alternatively, a suitable image can be exported and ROIs placed using proprietary software or public domain programmes. Significant correlation between histological steatosis and the SHRI has been found in several studies. In addition, point estimates of SHRI for the prediction of steatosis grades less than moderate or severe appear to be superior to those of qualitative grading methods [29]. The sensitivity and specificity of SHRI varied in some studies between $93-100 \%$ and $54-93 \%$ respectively [61-63].

The major limitation of this approach is the use of a reference system (kidney), whose echogenicity may also be altered by intrinsic illnesses. On the other hand, the parameter to be quantified in these cases is liver echogenicity and, respectively, kidney echogenicity. Since the hepatic echogenicity is not only the result of steatosis, but also of superimposed fibrosis, an altered renal parenchymal echotexture with renal parenchymal disorders may affect in this case US evaluations of the liver [28]. Moreover, the presence of hepatic fibrosis in some patients makes the linear correlation between fatty infiltration and liver echogenicity unreliable [27].

Another approach would involve the ultrasound attenuation coefficient calculated in the US image $[57,64]$. In a group of biopsied patients with hepatitis $\mathrm{C}$ infection, 
this coefficient was independently influenced only by steatosis, not by fibrosis or inflammation, and predicted mild steatosis and respectively moderate/severe steatosis with an AUROC of 0.734 and 0.842 , respectively [64].

\section{Noninvasive evaluation of liver steatosis using the Controlled Attenuation Parameter (CAP)}

Knowing that fat interferes with ultrasound propagation, a novel attenuation parameter has been developed to detect and quantify liver steatosis. It is called the controlled attenuation parameter (CAP). This ultrasonic attenuation coefficient is an estimate of the total ultrasonic attenuation (go-and-return path) at the central frequency of the regular or M Fibroscan ${ }^{\circledR}$ probe, and is expressed in $\mathrm{dB} / \mathrm{m}$. CAP is evaluated using the same radio-frequency data and the same region of interest, as the region used to assess the liver stiffness for fibrosis prediction [65-67].

In a recent meta-analysis [68] assessing the CAP accuracy for steatosis detection, the summarized sensitivity and specificity values were $0.78(95 \%$ confidence interval [CI], 0.69-0.84) and 0.79 (95\% CI, 0.68-0.86) for steatosis involving $<10 \%$ of hepatocytes, 0.85 (95\% CI, 0.74-0.92) and 0.79 (95\% CI, 0.71-0.85) for $11-33 \%$ fatty load, and 0.83 (95\% CI, 0.76-0.89) and 0.79 (95\% CI, $0.68-0.87$ ) for $67-100 \%$ fatty load. The median optimal cut-off value of CAP was $232.5 \mathrm{~dB} / \mathrm{m}$, and the values ranged from $214-289 \mathrm{~dB} / \mathrm{m}$ (for $<10 \%$ fatty load), 255 $\mathrm{dB} / \mathrm{m}$, with a range of $233-311 \mathrm{~dB} / \mathrm{m}$ (for $11-33 \%$ fatty load), respectively $290 \mathrm{~dB} / \mathrm{m}$, with a range of $266-318$ $\mathrm{dB} / \mathrm{m}$ (for $67-100 \%$ fatty load).

In comparison to other modalities, CAP is non-invasive, quantitative, and non-ionising. Furthermore, the procedure is easy to perform and provides immediate results; it is also machine-independent and does not require corrections to be made for gain, frequency, focusing or beam diffraction, and is also not subject to operator interpretation [65]. Compared to a liver biopsy, CAP is less prone to sampling error as it explores a liver volume $\approx 100$ times larger [66]. CAP can be performed simultaneously to liver stiffness measurement and in the same liver volume, making possible the simultaneous evaluation of both fibrosis and steatosis [69].

However, other prospective studies are mandatory before recommending this technique and the proposed cut-offs for the prediction of steatosis grades.

In conclusion, ultrasonography is an established tool used as a screening modality with acceptable sensitivity and specificity in detecting fatty liver. Nevertheless, the drawbacks of this technique include its inaccuracy in differentiating fibrosis from steatosis, in reproducibility and in the exact quantification of fat accumulation. Using the computerized analysis of US images and especially the new elastographic techniques seem to increase the performance of steatosis detection using ultrasounds.

\section{Aknowledgement}

This paper was published under the frame of European Social Found, Human Resources Development Operational Programme 2007-2013, project no. POSDRU/159/1.5/S/138776.

\section{Conflict of interest: none}

\section{References}

1. Lupsor M, Badea R. Imaging diagnosis and quantification of hepatic steatosis: is it an accepted alternative to needle biopsy? Rom J Gastroenterol 2005; 14: 419-425.

2. Younossi ZM, Stepanova M, Afendy M, et al. Changes in the prevalence of the most common causes of chronic liver diseases in the United States from 1988 to 2008. Clin Gastroenterol Hepatol 2011; 9: 524-530.

3. Lee SS, Park SH, Kim HJ, et al. Non-invasive assessment of hepatic steatosis: Prospective comparison of the accuracy of imaging examinations. J Hepatol 2010; 52: 579585.

4. Adams LA, Sanderson S, Lindor KD, Angulo P. The histological course of nonalcoholic fatty liver disease: a longitudinal study of 103 patients with sequential liver biopsies. J Hepatol 2005; 42: 132-138.

5. Farrell GC, Larter CZ. Nonalcoholic fatty liver disease: from steatosis to cirrhosis. Hepatology 2006; 43: S99-S112.

6. Starley BQ, Calcagno CJ, Harrison SA. Nonalcoholic fatty liver disease and hepatocellular carcinoma: a weighty connection. Hepatology 2010; 51: 1820-1832.

7. Leandro G, Mangia A, Hui J, et al. Relationship between steatosis, inflammation, and fibrosis in chronic hepatitis $\mathrm{C}$ : a meta-analysis of individual patient data. Gastroenterology 2006; 130: 1636-1642.

8. Poynard T, Ratziu V, McHutchison J, et al. Effect of treatment with peginterferon or interferon alfa- $2 b$ and ribavirin on steatosis in patients infected with hepatitis C. Hepatology 2003 ; 38 : 75-85.

9. de Meijer VE, Kalish BT, Puder M, Ijzermans JN. Systematic review and meta-analysis of steatosis as a risk factor in major hepatic resection. Br J Surg 2010; 97: 1331-1339.

10. Minervini MI, Ruppert K, Fontes P, et al. Liver biopsy findings from healthy potential living liver donors: reasons for disqualification, silent diseases and correlation with liver injury tests. J Hepatol 2009; 50: 501-510.

11. Nagai S, Fujimoto Y, Kamei H, Nakamura T, Kiuchi T. Mild hepatic macrovesicular steatosis may be a risk factor for hyperbilirubinaemia in living liver donors following right hepatectomy. Br J Surg 2009; 96: 437-444. 
12. Limanond P, Raman SS, Lassman C, et al. Macrovesicular hepatic steatosis in living related liver donors: correlation between CT and histologic findings. Radiology 2004; 230: 276-280.

13. Selzner M, Clavien PA. Fatty liver in liver transplantation and surgery. Semin Liver Dis 2001; 21: 105-113.

14. Chitturi S, Farrell GC, George J. Non-alcoholic steatohepatitis in the Asia-Pacific region: future shock? J Gastroenterol Hepatol 2004; 19: 368-374.

15. Grigorescu M, Rusu M. Non-alcoholic fatty liver disease, component of the metabolic syndrome: prevalence, profile of the risk factors. Roumanian Journal of Hepatology 2005; 1 (Suppl 1): 13-16.

16. Vilgrain V, Ronot M, Abdel-Rehim M, et al. Hepatic steatosis: a major trap in liver imaging. Diagn Interv Imaging 2013; 94: 713-727.

17. Angelico F, Del Ben M, Conti R, et al. Insulin resistance, the metabolic syndrome, and nonalcoholic fatty liver disease. J Clin Endocrinol Metab 2005; 90: 1578-1582.

18. Joy D, Thava VR, Scott BB. Diagnosis of fatty liver disease: is biopsy necessary? Eur J Gastroenterol Hepatol 2003; 15: 539-543.

19. Rockey DC, Caldwell SH, Goodman ZD, Nelson RC, Smith AD; American Association for the Study of Liver Diseases. Liver biopsy. Hepatology 2009; 49: 1017-1044.

20. Vuppalanchi R, Unalp A, Van Natta ML, et al. Effects of liver biopsy sample length and number of readings on sampling variability in nonalcoholic Fatty liver disease. Clin Gastroenterol Hepatol 2009; 7: 481-486.

21. Ratziu V, Charlotte F, Heurtier A, et al. Sampling variability of liver biopsy in nonalcoholic fatty liver disease. Gastroenterology 2005; 128: 1898-1906.

22. Schwenzer NF, Springer F, Schraml C, Stefan N, Machann $\mathrm{J}$, Schick F. Non-invasive assessment and quantification of liver steatosis by ultrasound, computed tomography and magnetic resonance. J Hepatol 2009; 51: 433-445.

23. Guiu B, Loffroy R, Hillon P, Petit JM. Magnetic resonance imaging and spectroscopy for quantification of hepatic steatosis: urgent need for standardization! J Hepatol 2009; 51: 1082-1083.

24. Hernaez R, Lazo M, Bonekamp S, et al. Diagnostic accuracy and reliability of ultrasonography for the detection of fatty liver: a meta-analysis. Hepatology 2011; 54: 1082-1090.

25. Goldberg B, Petterson H. The Nicer Year Book 1996 Ultrasonography, The Nicer Institute, ISIS Medical Media 1996: 55-81.

26. Badea R. Ficatul. In: Badea RI, Dudea SM, Mircea PA, Stamatian F. Tratat de Ultrasonografie Clinică. Ed. Medicală, Bucureşti, 2000: 105-176.

27. Mathiesen UL, Franzen LE, Aselius H, et al. Increased liver echogenicity at ultrasound examination reflects degree of steatosis but not of fibrosis in asymptomatic patients with mild/moderate abnormalities of liver transaminases. Dig Liver Dis 2002; 34: 516-522.

28. Chen CL, Cheng YF, Yu CY, et al. Living donor liver transplantation: the Asian perspective. Transplantation 2014; 97 Suppl 8: S3.
29. Gerstenmaier JF, Gibson RN. Ultrasound in chronic liver disease. Insights Imaging 2014 May 24. DOI 10.1007/ s13244-014-0336-2.

30. Mottin CC, Moretto M, Padoin AV, et al. The role of ultrasound in the diagnosis of hepatic steatosis in morbidly obese patients. Obes Surg 2004; 14: 635-637.

31. Dasarathy S, Dasarathy J, Khiyami A, Joseph R, Lopez R, McCullough AJ. Validity of real time ultrasound in the diagnosis of hepatic steatosis: a prospective study. J Hepatol 2009; 51: 1061-1067.

32. Lee JY, Kim KM, Lee SG, et al. Prevalence and risk factors of non-alcoholic fatty liver disease in potential living liver donors in Korea: a review of 589 consecutive liver biopsies in a single center. J Hepatol 2007; 47: 239-244.

33. Palmentieri B, de Sio I, La Mura V, et al. The role of bright liver echo pattern on ultrasound B-mode examination in the diagnosis of liver steatosis. Dig Liver Dis 2006; 38: 485489.

34. Wang CC, Hsieh TC, Tseng TC, et al. Factors affecting the diagnostic accuracy of ultrasonography in assessing the severity of hepatic steatosis. J Formos Med Assoc 2014; 113: 249-254.

35. Tobari M, Hashimoto E, Yatsuji S, Torii N, Shiratori K. Imaging of nonalcoholic steatohepatitis: advantages and pitfalls of ultrasonography and computed tomography. Intern Med 2009; 48: 739-746.

36. Saadeh S, Younossi ZM, Remer EM, et al. The utility of radiological imaging in nonalcoholic fatty liver disease. Gastroenterology 2002; 123: 745-750.

37. Brunt EM. Nonalcoholic steatohepatitis: definition and pathology. Semin Liver Dis 2001; 21: 3-16.

38. Strauss S, Gavish E, Gottlieb P, Katsnelson L. Interobserver and intraobserver variability in the sonographic assessment of fatty liver. AJR Am J Roentgenol 2007; 189: 320-323.

39. Oguzkurt L, Yildirim T, Torun D, Tercan F, Kizilkilic O, Niron EA. Hepatic vein Doppler waveform in patients with diffuse fatty infiltration of the liver. Eur J Radiol 2005; 54 : 253-257.

40. Bolondi L, Li Bassi S, Gaiani S, et al. Liver cirrhosis: changes of Doppler waveform of hepatic veins. Radiology 1991; 178: 513-516.

41. Mihmanli I, Kantarci F, Yilmaz MH, et al. Effect of diffuse fatty infiltration of the liver on hepatic artery resistance index. J Clin Ultrasound 2005; 33: 95-99,

42. Karcaaltincaba M, Akhan O. Imaging of hepatic steatosis and fatty sparing. Eur J Radiol 2007; 61: 33-43.

43. Basaran C, Karcaaltincaba M, Akata D, et al. Fat-containing lesions of the liver: cross-sectional imaging findings with emphasis on MRI. AJR Am J Roentgenol 2005; 184: 1103-1110.

44. Prasad SR, Wang H, Rosas H, et al. Fat-containing lesions of the liver: radiologic-pathologic correlation. Radiographics 2005; 25: 321-331.

45. Mortele KJ, Stubbe J, Praet M, et al. Intratumoral steatosis in focal nodular hyperplasia coinciding with diffuse hepatic steatosis: CT and MRI findings with histologic correlation. Abdom Imaging 2000; 25: 179-181. 
46. Khalili K, Lan FP, Hanbidge AE, Muradali D, Oreopoulos DG, Wanless IR. Hepatic subcapsular steatosis in response to intraperitoneal insulin delivery: CT findings and prevalence. AJR Am J Roentgenol 2003; 180: 1601-1604.

47. Valls C, Iannacconne R, Alba E, et al. Fat in the liver: diagnosis and characterization. Eur Radiol 2006; 16: 2292-2308.

48. Tchelepi H, Ralls PW, Radin R, Grant E. Sonography of diffuse liver disease. J Ultrasound Med 2002; 21: 1023 1032.

49. Itai Y. Peritumoral sparing of fatty liver: another important instance of focal sparing caused by a hepatic tumor. AJR Am J Roentgenol 2000; 174: 868-870.

50. Aubin B, Denys A, Lafortune M, Dery R, Breton G. Focal sparing of liver parenchyma in steatosis: role of the gallbladder and its vessels. J Ultrasound Med 1995; 14: 77-80.

51. Matteoni CA, Younossi ZM, Gramlich T, Boparai N, Liu YC, McCullough AJ. Nonalcoholic fatty liver disease: a spectrum of clinical and pathological severity. Gastroenterology 1999; 116: 1413-1419.

52. Taylor KJ, Gorelick FS, Rosenfield AT, Riely CA. Ultrasonography of alcoholic liver disease with histological correlation. Radiology 1981;141:157-161.

53. Meek DR, Mills PR, Gray HW, Duncan JG, Russell RI, McKillop JH. A comparison of computed tomography, ultrasound and scintigraphy in the diagnosis of alcoholic liver disease. Br J Radiol 1984; 57:23-27.

54. Yeh WC, Huang SW, Li PC. Liver fibrosis grade classification width B-mode ultrasound. Ultrasound Med Biol 2003;29:1229-1235.

55. Lin T, Ophir J, Potter G. Correlation of ultrasonic attenuation with pathologic fat and fibrosis in liver disease. Ultrasound Med Biol 1988; 14: 729-734.

56. Roldan-Valadez E, Favila R, Martinez-Lopez M, Uribe M, Mendez-Sanchez N. Imaging techniques for assessing hepatic fat content in nonalcoholic fatty liver disease. Ann Hepatol 2008; 7: 212-220.

57. Gaitini D, Baruch Y, Ghersin E, et al. Feasibility study of ultrasonic fatty liver biopsy: texture vs. attenuation and backscatter. Ultrasound Med Biol 2004; 30: 1321-1327.

58. Gaitini D, Lederman M, Baruch Y, et al. Computerised analysis of liver texture with correlation to needle biopsy. Ultraschall Med 2005; 26: 197-202.

59. Fujii Y, Taniguchi N, Itoh K, et al. A new method for attenuation coefficient measurement in the liver. Comparison with the spectral shift central frequency method. J Ultrasound Med 2002; 21: 783-788.

60. Osawa H, Mori Y. Sonographic diagnosis of fatty liver using a histogram technique that compares liver and renal cortical echo amplitudes. J Clin Ultrasound 1996; 24: 2529.

61. Marshall RH, Eissa M, Bluth EI, Gulotta PM, Davis NK. Hepatorenal index as an accurate, simple, and effective tool in screening for steatosis. AJR Am J Roentgenol 2012; 199: 997-1002.

62. Borges VF, Diniz AL, Cotrim HP, Rocha HL, Andrade NB. Sonographic hepatorenal ratio: a noninvasive method to diagnose nonalcoholic steatosis. J Clin Ultrasound 2013; 41: 18-25.

63. Webb M, Yeshua H, Zelber-Sagi S, et al. Diagnostic value of a computerized hepatorenal index for sonographic quantification of liver steatosis. AJR Am J Roentgenol 2009; 192: 909-914.

64. Lupşor M, Badea R, Vicaş C, et al. Detection of steatosis in chronic hepatitis $\mathrm{C}$, based on the evaluation of the attenuation coefficient computed on the ultrasound image. Med Ultrason 2008; 10: 13-20.

65. Sasso M, Beaugrand M, de Ledinghen V, et al. Controlled attenuation parameter (CAP): a novel $\mathrm{VCTE}^{\mathrm{TM}}$ guided ultrasonic attenuation measurement for the evaluation of hepatic steatosis: preliminary study and validation in a cohort of patients with chronic liver disease from various causes. Ultrasound Med Biol 2010; 36: 1825-1835.

66. Sasso M, Miette V, Sandrin L, Beaugrand M. The controlled attenuation parameter (CAP): a novel tool for the non-invasive evaluation of steatosis using Fibroscan. Clin Res Hepatol Gastroenterol 2012; 36: 13-20.

67. Sasso M, Tengher-Barna I, Ziol M, et al. Novel controlled attenuation parameter for noninvasive assessment of steatosis using Fibroscan( $\left({ }^{\circledR}\right)$ : validation in chronic hepatitis C. J Viral Hepat 2012; 19: 244-253.

68. Shi KQ, Tang JZ, Zhu XL, et al. Controlled attenuation parameter for the detection of steatosis severity in chronic liver disease: a meta-analysis of diagnostic accuracy. J Gastroenterol Hepatol 2014; 29: 1149-1158.

69. de Lédinghen V, Vergniol J, Foucher J, Merrouche W, le Bail B. Non-invasive diagnosis of liver steatosis using controlled attenuation parameter (CAP) and transient elastography. Liver Int 2012; 32: 911-918. 